\title{
Predicting Decision Propagation in Complex Sociotechnical System
}

This paper was downloaded from TechRxiv (https://www.techrxiv.org).

\section{LICENSE}

CC BY-NC-SA 4.0

SUBMISSION DATE / POSTED DATE

07-01-2021 / 09-01-2021

\section{CITATION}

Hassannezhad, Mohammad; Cassidy, Steve; Clarkson, P. John (2021): Predicting Decision Propagation in Complex Sociotechnical System. TechRxiv. Preprint. https://doi.org/10.36227/techrxiv.13536485.v1

DOI 


\title{
Predicting Decision Propagation in Complex Sociotechnical System
}

\author{
Mohammad Hassannezhad ${ }^{1,2, *}$, Steve Cassidy ${ }^{3}$, P. John Clarkson ${ }^{2}$ \\ ${ }^{1}$ Department of Automatic Control and Systems Engineering, University of Sheffield, United Kingdom \\ ${ }^{2}$ Engineering Design Centre, Department of Engineering, University of Cambridge, United Kingdom \\ ${ }^{3}$ Future Organizations Lab, British Telecommunications, Ipswich, United Kingdom
}

\begin{abstract}
Making informed decisions in today's ever more connected organizations requires a proactive approach to quantify the desirability of its possible consequences. A prominent problem within this realm is that the consequence of an individual's decision might propagate far beyond its local impact and globally affect multiple decisions and the entire system, often with amplifying and overlapping sphere of influences. Despite numerous efforts, the algorithmic complexity of overlapping propagation paths in a dense network is not resolved yet. This paper presents the development of a new computational model to tackles this problem from the window of change prediction. The proposed method, called Decision Propagation Method, aims to direct organizational decisions towards the most efficient set of sociotechnical interventions through predicting the risk of concurrent decision propagations. Designed on a case study in the Operations Engineering Group in British Telecom, it is illustrated that the proposed method, supported by an interactive prototype tool, offers a dynamic change prediction at a far less computational complexity and a better reproducibility which also requires less domain Knowledge from the experts.
\end{abstract}

Keywords: Change propagation; Complex networks; Computational intelligence; Decision-making; Engineering systems design; Interconnected Systems; Participatory modelling.

\section{Introduction}

Today's organizations are witnessing a growing complexity in making effective decisions. New connectivity technologies (such as knowledge-sharing platforms) have transformed the way individuals communicate and influence one another in a dynamic environment. As the result, an upstream decision might affect several consequences concurrently, while each of which might propagate beyond its local impact and globally affect multiple downstream decisions, sometimes without the influenced decision-makers necessarily taking any control over the situation [1-2]. Anticipating the collective risk of consequences in such situations is extremely difficult, and the knowledge of which can infer many implications across organizations towards a better understanding of the emergent system behavior, for example through constructing an individual feedback mechanism for each role to steer a better system performance. This paper tackles this challenge from the view of Engineering Change Management (ECM) [3].

By taking this view, a prominent problem is that increasing the degree of connectivity within and between system layers (or domains) directly affect the dispersion of Change Propagation (CP) [4], resulting into a proliferation of CP paths with, in many cases, amplifying, recursive (or cyclical), concurrent, and overlapping effects among them $[2,3,4,5]$.

\footnotetext{
*The corresponding author. Email address: m.hassannezhad@sheffield.ac.uk.
} 
Effective management of such situations requires the landscape of CP modelling to be expanded not only to handle the algorithmic complexity of a tightly connected (or dense) sociotechnical interfaces, but also take account of the recursive, concurrent, and overlapping effects into computation. Despite the huge repository of change prediction models, to the authors' knowledge, no previous research existed to provide such an integrated platform.

This paper describes the research that led to the development of Decision Propagation Method (DPM): a predictive $\mathrm{CP}$ method that is developed in response to the research gaps and the needs of industry sponsors. DPM considers an organization (and in general, a system) as a multi-layer network of individual decision-makers (as agents), decision variables, and possible outcomes and targets (as consequences), aiming to identify the most efficient sets of interventions in the organization through computing the collective risks between interfacing components, i.e., what settings at the decision layer achieve the minimum mutual risk between total cost and customer satisfaction (at the consequence layer), and what are the associated individuals (at the agent layer)? To support the implementation of the method, a computer support prototype is developed to link the interactive visualization with the underlying dynamic change model in such a way that encourages decision-makers to see the consequence of their actions in a real-time manner.

This paper is organized as follows. Section 2 describes the methodology behind this research and is followed by identifying the business requirements in Section 3. Section 4 outlines the previous research on CP. The proposed method is described in Section 5, with its implementation and analysis at the BT Group plc presented in Section 6. Eventually, the paper concludes in Section 7.

\section{Research Methodology}

This research began with framing the problem, through understanding the business challenges in the British Telecommunication Group plc (BT). The primary workshops, involving the cross-functional team of experts from Organizational Design, Field Operations, and OpenReach units, was necessitated the need for paying more attention to the dynamics of decision-making process, by highlighting the interplay between key decisions (and not every decision), influential decision-makers, and potential consequences. A fundamental hypothesis was framed as the result that is: an explicit understanding of the dynamics of connectivity between decision-makers, decisions, and consequences provides initiatives for cost-benefit analysis of interventions, resulting into improved system architecture.

Consolidation of the knowledge elicited from the workshops and from studying the company documents resulted into the development of the conceptual system map (Fig. 1). This knowledge, which was evolved over a time period of six months in a participatory process, was then used for benchmarking the solution concepts. The objective of benchmarking was to understand aspects of decision-making that are more important to company users (for example, individuals' behavior, decisions, connectivity) and then match them with advancements in the relevant literature in order to develop a proposal with a right focus at the right level of granularity, based on practitioners' feedback. The outcome was a range of alternative models developed to address the outlined business challenges (in Sec. 3) such as the concepts presented in [8] and [1].

The former model was based on the premise that one way to predict a system's performance is to focus on the changeability of its most-risky components. Therefore, the widely-used Change Prediction Method (CPM) [9] was used to identify the risky components and combined with a real-time System Dynamics model to interactively examine how dynamics of those components affect the key performance indicators. In the latter research, a conceptual decision analysis model was developed, based on the Bayesian chain rule, in that the proportional strength of the connectivity between decisions used to compute the Cumulative Confidence Ratio [1]. A primary case within the BT's Field Engineering Division (FED) was considered to assess the plausibility of the proposed concepts. Further investigation 


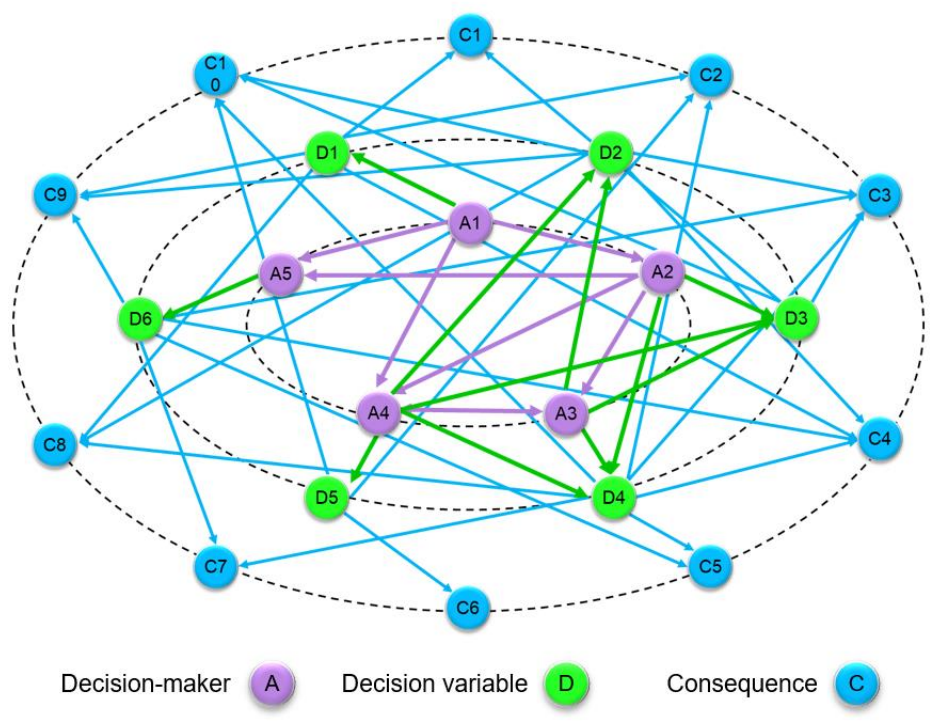

Fig. 1. Organization's decision-making can be seen a multilayer network of decision-makers, decisions, and their consequences, with interconnectivity.

of the business requirements during concept development highlighted a more fundamental set of questions reflecting the hypothesis, relating to:

- How to build a more composite view of decision network by mapping the semantic knowledge about the intra- and inter-connectivity into the model?

- How to quantify indirect influence of decisions in a multi-layer network by populating a compound risk diagram?

- What is most efficient set of interventions to make in the organization to achieve the desired set of outcomes such as mitigating the risk of unintended consequences (the outer ring in Fig. 1)?

Eventually, continuous refinement of the prototype models, over a time period of one year, led to the formulation of the DPM algorithm. The rest of this paper describes the steps towards formulation and implementation of the DPM in the BT's FED case.

\section{The Business Challenge}

Define As belong to one of the largest telecommunications infrastructures in the world, the FED is responsible for the forecasting, planning, and allocation of tasks for a network of nearly 25000 field engineers so as to enhance the quality of service and meet customer commitments while moderating the operations cost. The service delivery process in FED involves numerous stakeholders (such as employees, engineers, contractors, and customers) whose behavior, in conjunction with the technical system (e.g., forecasting models, field operations equipment) would not be the same on any two consecutive days. Given this complexity, satisfying objectives would not be possible unless building the capability to effectively manage foreseen and unforeseen changes. Change, in the FED, might originate from a variety of internal (e.g., employee) and external (e.g., weather, customer) sources; they are quite frequent and typically occur concurrently. For example, when some customers may not be at home (to perform a job) and some engineers may not meet the expected productivity (on delivering a job) on a specific day. The emergent impact of these changes, exemplified in Fig. 2, can cause significant problem for all stakeholders. 


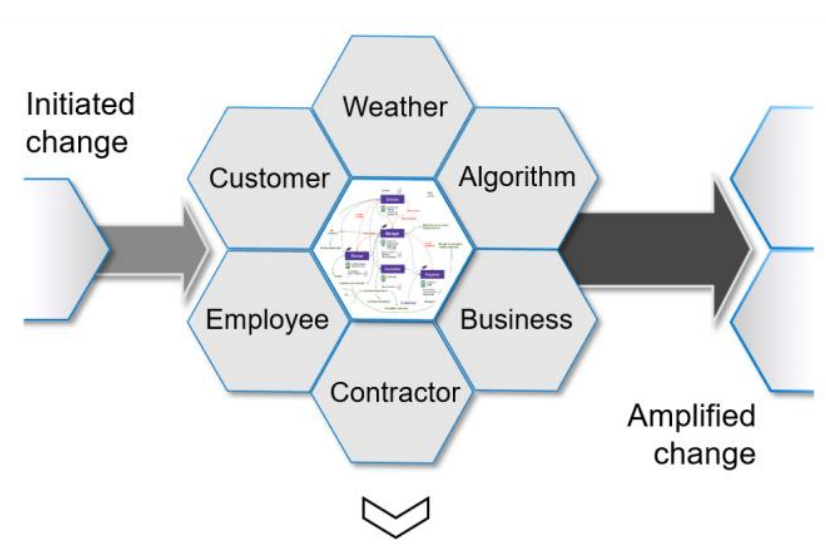

\begin{tabular}{ll}
\hline \hline Source & What can possibly change or go wrong? \\
\hline Weather & - Unexpected events such as the storm \\
Algorithm & - Forecasting model may be wrong \\
& - Uncertainty may be more than the thresholds \\
Business & - New products may be introduced \\
& - Priorities and targets may be changed \\
Contractors & - Service provider may make extra demand \\
& - Regulator may make mandates \\
Employee & - Engineers may not meet the expected \\
& - Engoductivity \\
& - Shineers may not accept overtime jobs \\
Customer & - Customer may change the requirements \\
& - Customer may cancel the jobs \\
& - Customer may not be at home
\end{tabular}

Fig. 2. Examples of sources of change in the FED case.
In this case study, there are five different roles belong to the different levels of organization, while each with a specific set of objectives and motivations as well as their own sources of pressure and varying degrees of interaction with other roles (agent layer in Fig. 1). Consider an instantiation of the system at the operational level. The Planners use a combination of a large historical dataset and weather forecasting models to produce a forecast of the amount of Repair tasks for the next 7 days. They are aware that any under-forecast (i.e., forecast of fewer tasks than actually occur) will very likely result in less engineers being deployed, thus resulting in SLAs ${ }^{2}$ being missed and the company incurring financial penalties. This shortfall can be mitigated by borrowing Engineers from contractors or asking the available Engineers to work overtime, but this contributes to diminish the productivity and worklife balance.

The decision about forecasting rate and Engineers' deployment consequently influence the Controllers, whose job is to assign the tasks to Engineers in a fashion that enable SLAs to be met at the end of the day. In order to compensate an under-forecast plan, Controllers might improve the productivity of Engineers by organizing the tasks so as to minimize the travelling time, but this could cause more tasks failing the SLA. The key challenge for Controllers is that they

have no control over how many tasks are allocated, how many engineers are deployed, or how many jobs an Engineer can perform per day.

This example shows, yet in a crude way, how performance of each role in FED can affect their own local consequences and the same outcome globally comply with the consequences of the other roles and the entire system. It is also demonstrated that when multiple changes are concurrently propagated through a common component, their impact cannot be considered independently; e.g., failing an SLA is a direct or indirect consequence of multiple decisions that are made by individuals of different roles. Due to the limited knowledge of each role on the other disciplines, making well-informed decisions in such situations is very difficult which requires each role to be proactively aware of a broader picture of the consequence propagation of its actions.

\subsection{Challenges in modelling the emergent behavior in FED}

The primary workshops at BT were identified the need for a predictive tool that can orchestrate opportunities for future improvement rather than troubleshooting the existing problem. Such as tool should be able to not only identify priorities (i.e., what components should be changed to achieve the desired outcome) but also consult on the dynamics

2 SLA, stands for Service Level Agreement, is a commitment between a service provider (BT, in our case) and a service receiver (customer), aiming to assure the delivery of an optimal service to customers, that contains a number of components to clearly define type of service that should be provided relating to the quality, availability, and responsibility of multiple parties. 


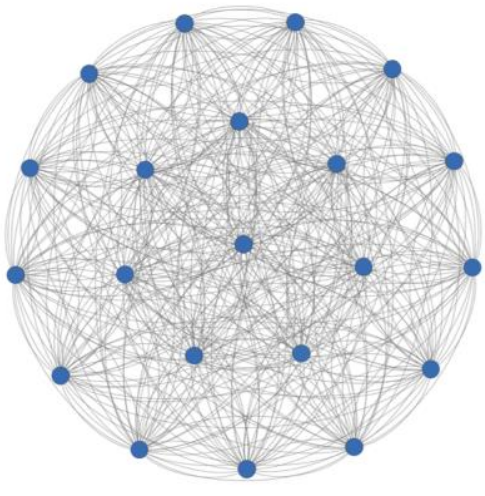

(a) Applying network analysis to the FED case to study change behavior of individual components

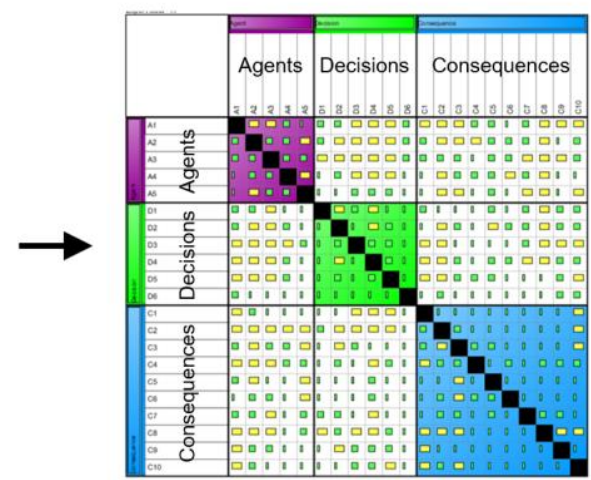

(b) Converting FED network into CPM risk matrix (in Cambridge Advanced Modeller) to study collective change behavior

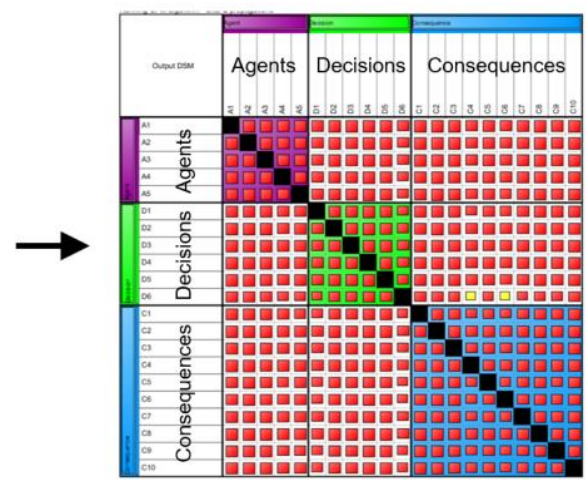

(c) Applying CPM algorithm with three propagation steps resulted into fully saturated risk matrix

Fig. 3. Examples of benchmarking solution concepts.

of interventions (i.e., what settings at these components are required to satisfy objectives). Accordingly, a range of approaches - including systems dynamics, network analysis, and CPM [9] - utilized to understand the collective risk of CP (Fig. 3), but the implications were limited in several aspects when populating the model. These limitations, grounded in further investigation of the business case with regards to the literature, are outlined in the following and established the foundation for development of a new method.

Sociotechnical interfaces. At the organizational level, effective management of changes requires incorporating the dynamics of the teams whose decisions affect successful implementation of these changes. This is evidently a critical task for managers [10] as it is very difficult to understand (and measure) how individuals behave and respond to different situations. Many communications are made through informal channels and many decisions are taken based on individual perceptions. Tracking all these channels and their emergent influence on the outcomes requires a model to be capable of not only dealing with the subjectivity of large amount of interface data, but also handling different types of coupling between them.

Density of connections. When expanding the modelling scope to a sociotechnical system, by highlighting the mechanism by which social network influence one another and the technical system (e.g., through knowledge-sharing platforms or organization's hierarchy), this will remarkably increase the degree of connectivity within and across layers [11]: in the FED case, resulting into a fully connected network out of workshops (Fig. 3(a)). This is a substantial problem for change modelling, as the most commonly used CP algorithms such as CPM [9] are based on a sparse matrix and cannot accommodate this density into the modelling. Fig. 3(c) shows an example of how increase in degree of connectivity of FED case made the outcome of CPM algorithm fully saturated.

Concurrency and overlapping impact. Changes do not occur in isolation often with amplifying and overlapping impact, but majority of the existing algorithms assume propagation of a single change at a time while no evidence found to be capable of accounting multiple CP paths with overlapping spheres of influences. In the example of FED case, earlier in this section, it is shown that the number of SLAs to be met is affected by changes in a number of factors passing through common individuals (e.g., Planner, Controllers, Engineers) whose propagation paths cannot be considered independently. 


\section{Change Propagation in the Literature}

If $\mathrm{A}$ fundamental assumption to prior research in modelling $\mathrm{CP}$ was that change in a network propagates via its direct connectivity with adjacent neighbors; therefore, the more connectivity going out of components, the higher degree of emergent propagation with nonadjacent components [3]. Two mainstreams of research have established as the result, with the focus on understanding (a) the Individual change behavior of system components (through analyzing the tendency of a component to propagate changes to the other components) and (b) the Collective change behavior (through analyzing the compound risk of propagation in the entire system). This categorization can be further divided into Matrix-based and Network-based analysis (from algorithmic standpoint) and Single-layer or Multi-layer models (from architectural standpoint). Fig. 4 displays some examples of prior research within each category. Note that this organizing framework is not exhaustive and only used to draw the boundary of this research. For overview of the relevant literature, the reader would refer to the references such as [12] and [13].

Routed in network science [39], the core objective of research on Individual Change Behavior has been the development of metrics to classify or rank network components. Such classification might infer several implications pertaining to for example the evaluation of modularity (e.g., [14], [20]), flexibility (e.g., [21], [40]), and susceptibility (e.g., [7], [22], [26]) of a system. Accordingly, there is a considerable evidence of using structural properties of complex networks in the literature, most notably aspects of Centrality and Betweenness metrics [20], [22], [23], [35]. Alongside, there has been some use of matrix properties such as Eigenvector [7] and Matrix Multiplications [14] and [27] to analyze the changeability of system components. While these studies can provide insightful information on propensity of components in influencing their adjacent or nonadjacent neighbors, they do not account the dynamics of CP across the system and majority of which do not accommodate the risk of propagation paths between pairs of (initiating and affected) components.

Another stream of research existed looking at Collective Change Behavior through quantifying the compound risk of propagation across the system when changes initiate from one or more components. Majority of these models are to some degree inspired or derived from the original CPM [9]. CPM is a probabilistic change prediction method that uses the product of likelihood (i.e., how likely a change in initiating component will lead to a change in affected component across their common interface) and impact (i.e., the average proportion of work required to adopt the propagated change), to quantify the combined (direct and indirect) risk of CP using a brute-force search algorithm.

The original CPM developed to quantify the risk of propagation between physical components of a product using a single-layer DSM. This has evolved over years to expand the scope of change modelling to multiple layers. For example, Hamraz et al. [28] applied the concept of Function-Behavior-Structure (FBS) in CPM and presented a linkage model to analyze the CP between layers. Koh et al. [4] developed the index of reachability to restrict the maximum length of CP paths in a multi-layer CPM to be examined. It was based on the premise that the combined probability of change diminishes as the CP path gets longer. Ahmad et al. [31] enhanced the functionality of CPM to consider multiple initiating changes and combined it with a graphical interface to facilitate tracking a change from product requirements through to the process task. Further examples are the least-risky CP model presented by Ullah et al. [18], workload-based analysis model by Tang et al. [19], tree-based structured language by Fdhila et al. [32], and approximated Bayesian model by Lee and Hong [34].

Likewise, there has been a growing attention in the literature to expand the measure of risk so as to additionally consider the impact of CP on high-level system properties such as duration and cost. For example, Chua and Hossain [16] utilize a transition matrix to compute the probability of change on downstream activities and combine it with a scheduling model to estimate the impact of CP on project completion. Li et al. [17] focus on sequential CP and present a mathematical algorithm to calculate the shortest propagation path between pair of components. The shortest path in their study refers to the propagation path that can fulfil the change requirements with the minimal impacts. Wynn 


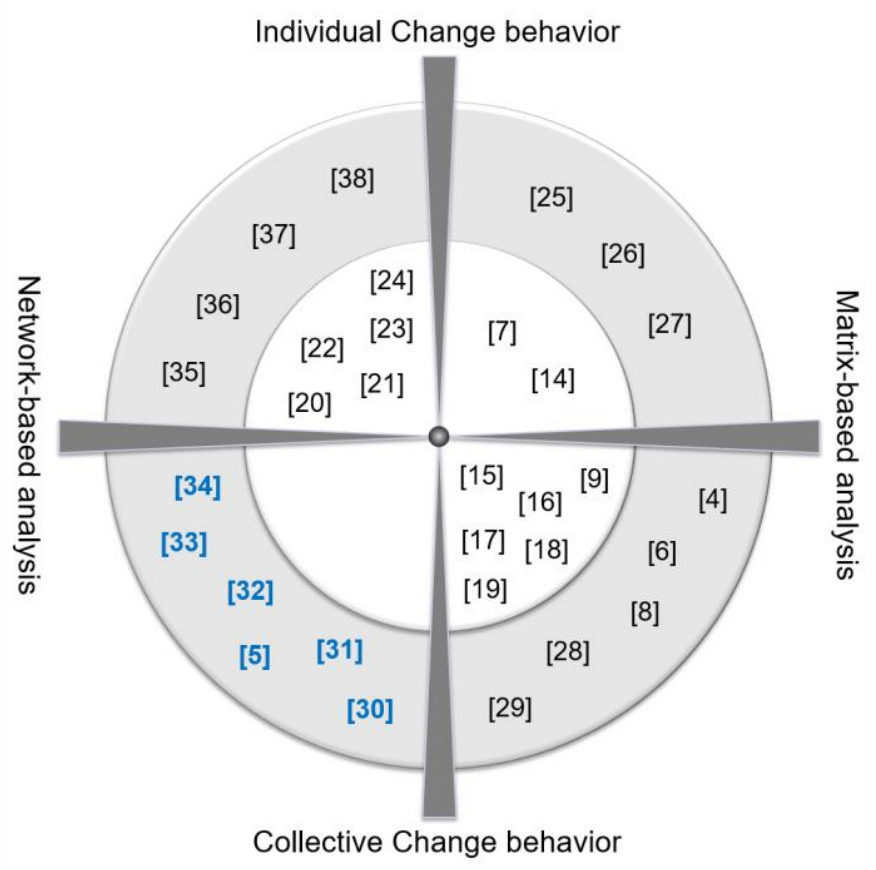

Fig. 4. An organizing framework of previous work on change propagation modelling: (1) the axes show the relative boundary between areas and are not absolute margins; (2) the inner and outer circles respectively represent the Single-layer and Multi-layer CP models (note: it refers to the main purpose for which the method has been developed and does not consider the potential for being extended to consider multiple domains; a famous example is the CPM which was primarily developed to analyze the risk of a product components in a single-domain DSM); (3) the shadowed area reflects the scope of this work; (4) the bluecolored references refer to the models that are capable of analyzing multiple initiating changes concurrently.

et al. [5] incorporate design changes in task input information and present a resource-sensitive simulation model to quantify the impact of changes on design process duration and resource.

\subsection{The need for a new approach}

Engineering change has not previously been studied as a purpose for anticipating the consequence of decisions in a complex organization with sociotechnical interfaces. Such an effort requires the term Decision Propagation to be understood as a Multi-layer Sociotechnical Change Propagation problem in that all types of coupling between components such as direct, indirect, cyclical, and overlapping impact are allowed. Accepting this view, the review of relevant literature in this section, influenced by BT challenges, shows that there is not a single model in the literature being capable to fulfil all the business requirements illustrated earlier in Sec. 3. In particular, our observations reveal a sufficient breath of research in change modelling, yet with a more emphasize on the theoretical enrichment of the concept rather than developing a more rigorous approach in dealing with real complex situations.

A key shortcoming of the literature is associated with the capability of models in handling tightly connected (or dense) networks. The research community typically cope with this challenge by simplifying the reality, for example through freezing the least-important links [41]. This can pose a danger when populating the change propagation, since an unimportant link might be a carrier of an important change across the network. This would be even more problematic in multi-layer networks, where each layer implies a different meaning, and the unimportant link might be an interfacing link which connect two different domains.

As far as related to the implementation of existing models, there is evidently a need for computer support to facilitate communication of the tool with decision-makers (i.e., enabling storyboarding and storytelling). The 


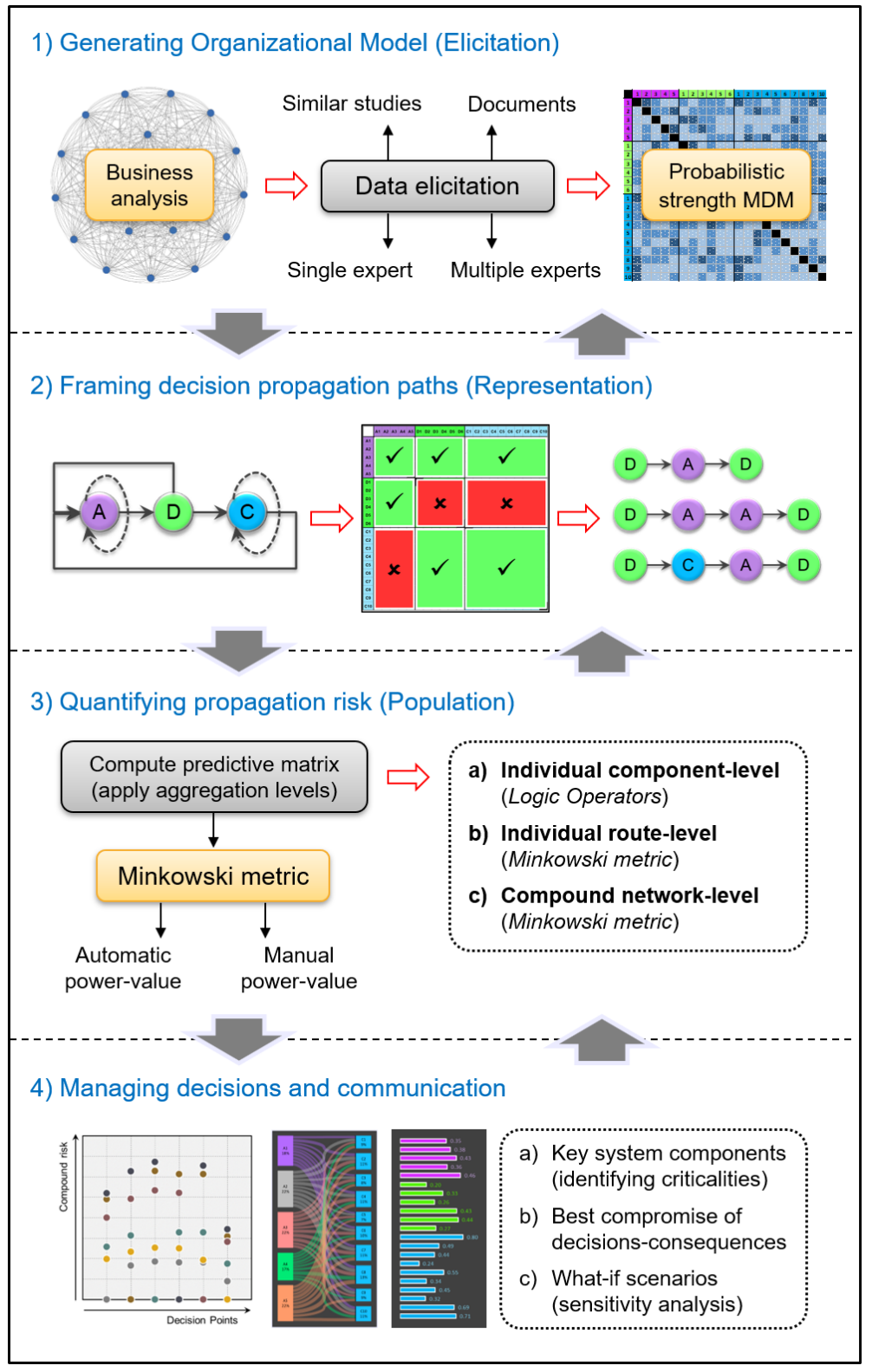

Fig. 5. Overview of the Decision Propagation Method.

importance of computer-based change support is well recognized within the research community [5][32] and for practitioners [42][10]: it provides ready access to the underlying change model in a way that enables the decisionmakers to understand, plan, implement, and communicate a change request. Few examples from the literature are the Change Modelling (or CPM) toolbox of the Cambridge Advanced Modeler (CAM) [41, p.28] - as the most commonly used non-commercial tool, DEPNET [30], C³ro Editor [32], RedesignIT [44], ADVICE [45], and Vatram [29].

Overall, the findings at this stage suggest that there is a need in both research and in industry for development of a new decision propagation tool to enable quantifying the collective risk of agents' decisions and their consequences in a complex organizational system. Such a tool should provide a flexible platform to guide practitioners towards finding the most efficient sets of interventions in the organization, while its underlying change algorithm is capable to accommodate sociotechnical interfaces, resolve the algorithmic complexity of a dense network, and handle multiple initiating changes with their overlapping impacts. 


\section{A Decision Propagation Method for Complex Sociotechnical system}

This section describes the proposed Decision Propagation Method (DPM): an alternative way of modelling CP that extends the current modelling paradigm focusing on interfaces within technical system to the intended sociotechnical interfaces.

\subsection{Overview of the Decision Propagation Method - DPM}

The DPM is a forward-looking approach to decision-making that dynamically seeks a better intervention between agents and their associated decisions by articulating the risk of consequences. It is based on the premise that, in a sociotechnical system, individuals make decisions and decisions possess consequences, which in turn affect the behavior of agents. Therefore, the desirability of a decision in such condition depends on the mutual risk between consequences, and the sensitivity that consequences show to a change in each decision and decision-maker. The measure of risk, in this context, might infer both positive and negative meanings: as it only determines the strength of change influence. The intent is to be proactive and to contemplate the direct and indirect consequences before attempting any action. Such analysis can therefore be used to not only deliver a dynamic set of decisional solutions at any given situation, but also improving the communication and collaborative decision-making amongst the team between which there may or may not be a direct link.

What distinguished DPM from the previous studies is (1) the incorporation of individuals' influences into decision analysis, (2) the capability to build an instantiation of the system and evaluate its performance with respect to the whole system behavior, and (3) the definition of risk, as the product of Likelihood and Impact, which incorporates both types of change behavior into the computation. Likelihood represents the 'collective change behavior' by obtaining the compound probability of CP between any pair of components. Impact reflects the 'individual change behavior' by quantifying the degree of centrality of a component with respect to its adjacent neighbors. Learning from the analysis of literature shows that such incorporation gives a more realistic and holistic view of system behavior when impact of a change propagates across multiple system layers. In particular, the measure of likelihood enables the user to govern multiple (often hidden) interdependencies between any two points in the system, and the measure of impact gives an approximation of the changeability of these points. The only input to the DPM algorithm is the proportional strength of the direct links between components which are represented in form of probability within the range of $[0,1]$.

Structurally, the method consists of four steps: generating organizational model (as a kind of causal-effect diagram), architecting decision propagation views, quantifying propagation risk, and managing decisions through visualization. These stages, shown in Fig. 5, are explained in the following sub-sections:

\subsection{Stage.1: Generating Organizational Model}

The method begins with analyzing the business case in order to obtain an initial organizational model. The outcome of this (ideally participatory) process is a probabilistic square multi-layer matrix (MDM) shown as $\|Y\|=\left(y_{i j}\right)_{p \times p}=$ $(A, D, C)_{p \times p}(\forall p=m+n+k)$ with three layers corresponding to the agents (individuals who make or assess decisions: $A=\left\{a_{1}, \ldots, a_{m}\right\}$ ), decisions (variables that vary according to agents' preferences: $D=\left\{d_{1}, \ldots, d_{n}\right\}$ ), and consequences (performance indicators reflecting objectives: $C=\left\{c_{1}, \ldots, c_{k}\right\}$ ), in that column headings show the initiating components and rows the influenced components.

The value of matrix in the organizational model is set to qualitatively represent the proportional strength of a change influence between initiating and affected components: it is delineated in form of high $(H)$ if the influence is significant, medium $(M)$ if it is moderate, and low $(L)$ if it is insignificant. Being used in a number of prior studies in the literature (such as in the references [15][46][47]), it was also the preferred way of capturing data uncertainty by practitioners, since acquiring the precise value of probabilities is not straightforward which requires significant 
numeracy of the case. This qualitative data, shown by color-coding in Fig. 5, is then equalized with the numerical values of $0.2,0.5$, and 0.8 to respectively represent the low, medium, and high levels of strength, when architecting the model.

\subsection{Stage 2: Framing decision propagation paths}

A resultant challenge for modelling the sociotechnical system interfaces is related to the inherent complexity in explicit understanding of the flow of influence within an organization: it seems to be much richer than what the initiating and affected agents suppose, and their emergent organizational model much denser [41]. During our subsequent workshops with practitioners, it is also discussed that the flow of influence might be different if architecting the entire organization or an instantiation of it.

An implication is that when, for example, mapping the organizational dynamics at a holistic network level, the direct influence between decision networks cannot be ignored, but by narrowing down the scope to a specific business situation, decisions act like a physical object which can only influence and being influenced through the decisionmakers. Furthermore, at the network level, the model contains so many branches (paths) that they cannot be fully independent of the others. Therefore, there must be some degree of commonality or overlap between network paths.

Therefore, the second stage in DPM is concerned with determining the focus of modelling. This will be accomplished by a set of two (complementary) pruning strategies to confine the number of expected propagation paths:

Static Pruning: carried out at the pre-population design time, as part of the matrix mapping configuration. This strategy directly applies the fundamental assumption of the modelling - that is agents make decisions, decisions influence consequences which in turn affect the agents' behavior - by neutralizing the corresponding boxes in the organizational MDM (Fig. 5, step 2);

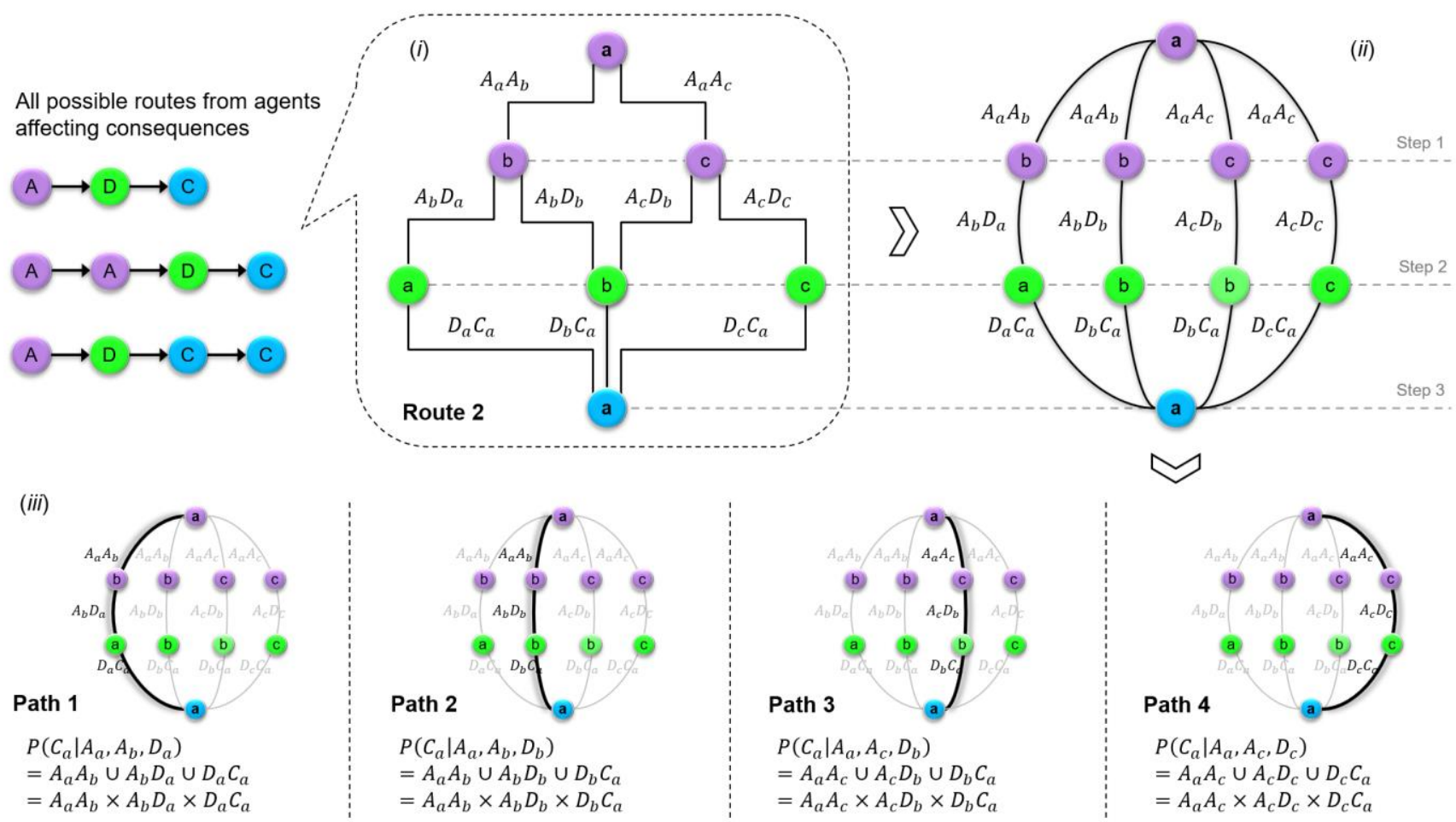

Fig. 6. Individual Component-level aggregation in DPM: example of a partial propagation tree. 
Dynamic Pruning: carried out at the post-population run time, when the propagation algorithm is run. For the purpose of this study, we prune all paths whose length exceeds three steps. Without increasing the computational complexity, this length number has been realized to be sufficient to track the flow of a change across (three) layers back to the original layer where the change was initiated, hence capturing the cyclical CP. In addition, when validating the method, the experts realized three propagation length to be surprising enough to make the results non-trivial while still specific enough without being lost of the input information (Sec. 6.1).

Placing the emphasis on propagation channels, this stage distinguishes the route-type towards affecting a component. This capability can be used to examine the criticality of a certain path, or the impact of a new decision-making strategy, e.g., what is the impact of delegating a decision to a different agent or making an individual decision a collaborative decision, by sharing the responsibility between multiple agents? (See Sec. 6.1) The outcome of this step is a three-step propagation tree that is generated between each pair of components for all possible routes - similar to Trail Counting [47, p.104] - such as the one illustrated in Fig. 6( $)$ from agent $A_{a}$ to consequence $C_{a}$. This propagation tree, counting for both direct and indirect dependencies, is then used to quantify the propagation risk.

\subsection{Stage 3: Quantifying propagation risk}

The Compound Risk $\left(C R_{a b}\right)$ of a change between initiating component $(a)$ and affected component $(b)$ is a product of Compound Likelihood and Impact, analogous to the CPM [9]:

Compound Risk $\left(C R_{a b}\right)=$ Compound Likelihood $\left(C L_{a b}\right) \times \operatorname{Impact}\left(I_{a}\right)$

Compound likelihood $\left(C L_{a b}\right)$ is an approximation of the probability that a change in an instigating component $(a)$ will lead to change in a nonadjacent component $(b)$. The key to such an approximation is the aggregation of change data across interfacing components so as can properly reflect the interdependencies between propagation paths. Impact of a change $\left(I_{a}\right)$ is a ratio that refers to the relative intensity of CP that an initiating component exerts on or receives from its adjacent neighbours. It is a measure for analyzing the changeability of an individual component that shows the behaviour of a component with respect to the entire system architecture).

\section{A) Computing the Compound Likelihood}

The Computing the $C L$ in DPM is a systematic three-step process, respectively counting for (a) vertical aggregation of direct links between common interfaces in a single path, (b) horizontal aggregation of multiple paths in a single route, and (c) horizontal aggregation of multiple routes in the network. The rationale is that people might react to decisions differently at various circumstances. This matters if an agent for example $A_{a}$ in Fig. 6( $\imath$ ) directly influence the decision $D_{b}$ or indirectly through influencing $A_{b}$ and $A_{c}$ : the result might not necessarily be the same. This varied behavior requires each single path in the network to be considered separately with step-wise aggregation to make sure not to lose important interface data at each level. These steps are explained in the following:

(a) Component-level aggregation. Considering a partial propagation tree, such as the one illustrated in Fig. 6 between $A_{a}$ and $C_{a}$, the algorithm views each propagation path independently (Fig. 6(iv)) and uses the AND (U) operator to combines the probability of common interfaces within each path. Comparing to CPM, in which the computation begins at the bottom farthest from the initiating component, the proposed algorithm starts from the top for each individual path. In fact, it is based on the concept of path searching in that if for example there is a link between $A_{a}$ and $A_{b}$, and $A_{b}$ can affect $D_{a}$, then $A_{a}$ can indirectly affect $D_{a}$ with a probability that is less than a direct link between them. Since the components are mutually exclusive, the formulation will take the form of: 


$$
P\left(C_{a} \mid A_{a}, A_{b}, D_{a}\right)=A_{a} A_{b} \cup A_{b} D_{a} \cup D_{a} C_{a}=A_{a} A_{b} \times A_{b} D_{a} \times D_{a} C_{a}
$$

(b) Route-level aggregation. When aggregating the interfacing components at the first step, there is an assumption about the independence of multiple paths that belong to the same route. However, this situation is very unlikely in reality since for instance, when there are two paths 3 and 4 in Fig. 6(iii) both involving the same agent $A_{c}$, their influence on $C_{a}$ cannot be fully independent of each other. This can accordingly contribute to an overlapping impact between these paths, where the degree of overlapping is positively correlated with the number of paths containing common interfaces.

This study discusses that when making a decision, if for example there are two components influencing an individual's decision, it is more likely that their propagation paths are independent; but if an individual's decision is influenced by multiple changes, it is more likely that their propagation paths are to some degree overlapped and there might be a dominant path amongst them. As the result, the more propagation path in-coming, the lower probability of aggregated path which implies a kind of consolidation of independence.

Given the combined probability of each path in the first step, this step aggregates multiple propagation paths of the same route. Mathematically, this can be regarded as computing the proximity between these probabilities, equivalent to measuring the overlap between probabilities as distance. Therefore, inspired by the generalization of distance metrics in an $n$-dimensional space, we found the Minkowski metric sufficiently agile to envisage a nonlinear approximation of the relativistic effects between $C L s$. In fact, it is the most commonly used proximity measure in graph theory, with numerous applications in the fields such as data mining and pattern recognition [49][50]. For a real number $p \in \mathbb{R}>0$ ( $p$ is the number of dimensions), the Minkowski measure of norm $p$ can defined as below:

$$
\|x\|_{p}=\left(\left|x_{1}\right|^{p}+\left|x_{2}\right|^{p}+\cdots+\left|x_{n}\right|^{p}\right)^{\frac{1}{p}}
$$

Where $\left|x_{i}\right|$ refers to the combined probability for each propagation path (as the outcome of the previous step), and $n$ refers to the number of propagation paths which may be or not equivalent to the power of formula $(1 \leq p \leq n)$. Accepting this view means that the more propagation path existed between initiating $\left(A_{a}\right)$ and affected $\left(C_{a}\right)$ components, the higher value of $p$ and accordingly, the lower probability of aggregated path (in the given route) which implies a kind of consolidation of independence between multiple paths (Fig. 7). For the given example in Fig. 6, the route-level aggregation would be equal to:

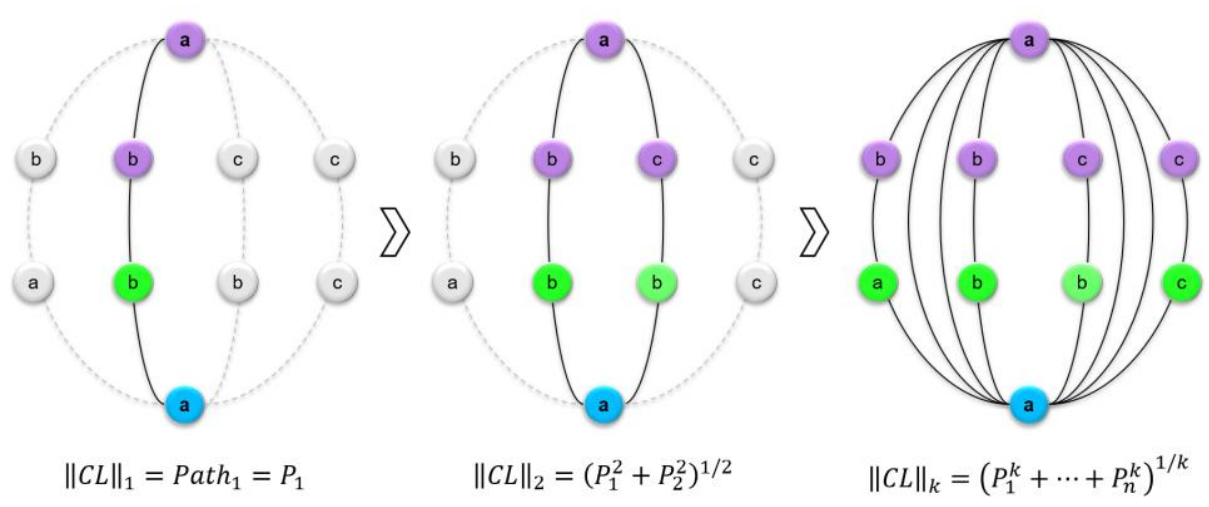

Fig. 7. Computing the overlapping dependency between propagation paths in DPM. 
$\left\|P_{\text {Route } .2}\right\|_{4}=\left(\sum_{k=1}^{4}\left|x_{\text {path }_{k}}\right|^{4}\right)^{\frac{1}{4}}=\left(\begin{array}{c}\left|P\left(C_{a} \mid A_{a}, A_{b}, D_{a}\right)\right|^{4}+\left|P\left(C_{a} \mid A_{a}, A_{b}, D_{b}\right)\right|^{4}+ \\ \left|P\left(C_{a} \mid A_{a}, A_{c}, D_{b}\right)\right|^{4}+\left|P\left(C_{a} \mid A_{a}, A_{c}, D_{c}\right)\right|^{4}\end{array}\right)^{\frac{1}{4}}$

(c) Network-level aggregation. By obtaining the aggregated probability of each route in the previous step, the algorithm then uses the same formula in (4) to aggregate multiple routes by which a change in component $A_{a}$ may propagate to the component $C_{a}$. However, the power of formula $(p)$ at this level will be confined within the range of 1 and the number of possible routes. The network-level aggregation for the example of Fig. 7 would be equal to:

$C L_{A_{a} C_{a}}=\left\|P_{\text {Network }}\right\|_{3}=\left(\sum_{n=1}^{3}\left|x_{n}\right|^{3}\right)^{\frac{1}{3}}=\left(\left(\sum_{i=1}^{3}\left|x_{A, D, C}\right|^{3}\right)^{3}+\left(\sum_{j=1}^{4}\left|x_{A, A, D, C}\right|^{4}\right)^{3}+\left(\sum_{k=1}^{2}\left|x_{A, D, C, C}\right|^{2}\right)^{3}\right)^{\frac{1}{3}}$

Therefore, the algorithm not only considers the interdependence between propagation paths that belong to the same type (e.g., $A \rightarrow D \rightarrow C$ ), it also counts for possible overlapping between different routes, for example taking the influence of communication between agents $(A \rightarrow A \rightarrow D \rightarrow C)$ or the mutual impact between consequences $(A \rightarrow$ $D \rightarrow C \rightarrow C$ ) into consideration. This multiple-level aggregation of probabilities might produce numbers greater than 1 , so the algorithm normalizes the $C L$ s values at the end of last step to ensure that they lie within the range of $(0,1)$. The final value will then be multiplied by impact $\left(I_{a}\right)$ to obtain the $C R_{a b}$.

\section{B) Computing the Impact}

When Routed in Network Science [39], the measure of impact in DPM represents the tendency of an initiating component $\left(I_{a}\right)$ to propagate changes to the other components. Semantically, it is comparable to the Change Propagation Index (CPI) presented in Giffin et al. [22] that is computed based on number of outgoing links minus incoming links. Similarly, Kreimeyer [51] enumerates the degree of correlation between number of outgoing and incoming links as a metric for understanding the degree to which a component can affect or being affected by other components. This paper introduces the measure of Criticality $(\mathrm{Cr})$ as the fraction of the cumulative probability of outgoing links (value of columns in organizational MDM) over incoming links (value of rows in organizational MDM):

Criticality $\left(\mathrm{Cr}_{i}\right)=\frac{\text { Weighted sum of outgoing links }}{\text { Weighted sum of incoming links }}$

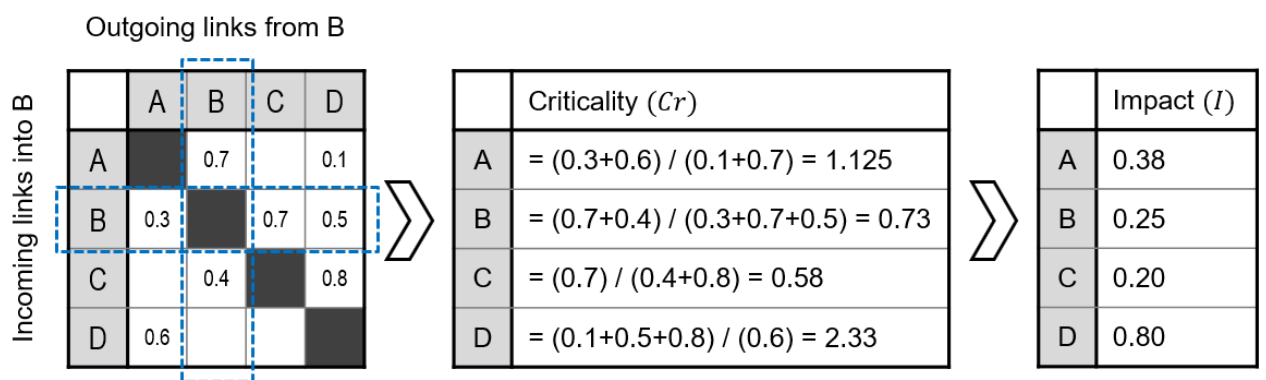

Fig. 8. Example of computing impact in DPM; the values are bounded within the range of $[0.2,0.8]$. 
Where $i$ refers to the initiating component. $C r_{i}$ can take any value within the range of $(0,1)$. It is based on the fundamentals of complex networks in that there is at least 1 outgoing link from and 1 incoming link to a component (with no isolated component in the network). In fact, it is a measure for analyzing the changeability of system components by looking at the intensity of changes that a component exerts on (outgoing activity) or receive from (incoming passivity) its adjacent neighbors. As $C r_{i}$ can take any positive value (see Fig. 8 for step-by-step process), Feature Scaling is then used to restrict the values between the arbitrary points of $a$ (lower strength bound) and $b$ (upper strength bound). This will eventually give us the impact, as the formula shows:

$\operatorname{Impact}\left(I_{i}\right)=\left(a+\frac{\left(C r_{i}-C r_{\min }\right) \times(b-a)}{\left(C r_{\max }-C r_{\min }\right)}\right)$

The measure of impact can be used as an approximation for analyzing or ranking system components. When combining with $C L_{i j}$, to produce the compound risk $C R_{i j}$, they can complement each other in such a way that $I_{i}$ looks at aptitude of a component in influencing others (if change occurs) and $C L_{i j}$ quantifies the magnitude of that influence on nonadjacent components (between which there is not a direct link). Therefore, a component with the greatest impact value might not necessarily be the riskiest one, in terms of its emergent influence on other components.

\subsection{Stage 4: Decision management}

The final stage in DPM is concerned with the communication of outputs, sensitivity analysis of inputs, and scrutinizing the system behavior to identify improvement leverages of system architecture for further iterations. Once the compound risk $\left(C R_{i j}\right)$ matrix has been derived, a variety of charts and diagrams may be used to visualize the resultant data. The primary outcome of the model would be an impact bar (identify most-least influential components) and a risk plot (identifying low-risk leverages). It can be tailored to add extra information regarding to for example multiple expert views, and the sensitivity that consequences show to agents' behavior or to different propagation paths.

The initial analysis can be used to create business narratives that help explain the model behaviours to the experts who provided data. This will provide a mechanism for the model user to identify most efficient interventions (e.g., by comparing parallel views simultaneously) and also getting the experts to re-evaluate their inputs. Therefore, as well as predicting sociotechnical change interfaces, the overall aim of quantitative analysis is to get the experts to the point where they agree on a specific improvement scenario. The following section outlines the implementation of DPM.

\section{Implementation and evaluation}

The implementation of DPM was an iterative process with regular calibration after each development phase over the course of six months. Totally, three (two full-day and one half-day) workshops arranged with key stakeholders and domain experts (including mathematician, engineers, and physicians) to verify usability of the tool and credibility of the results. The requirements of a decision support tool for hosting at BT, listed in Table 1, was previously determined during the benchmarking workshops and refined during the literature analyses.

As the result, a computer support was developed using Visual Basic programming language in Microsoft Excel (Fig. 9) to be compatible and portable across operating systems. The DPM dashboard has been tailored in such a way that embraces the challenges that have been determined during the workshops with partners (Req. \#15), with placing the graphical emphasize on the mutual influence between consequences and the sensitivity that consequences show to each agent and decision. To ensure that users can perform real-time decision analysis without having to code (Req. 


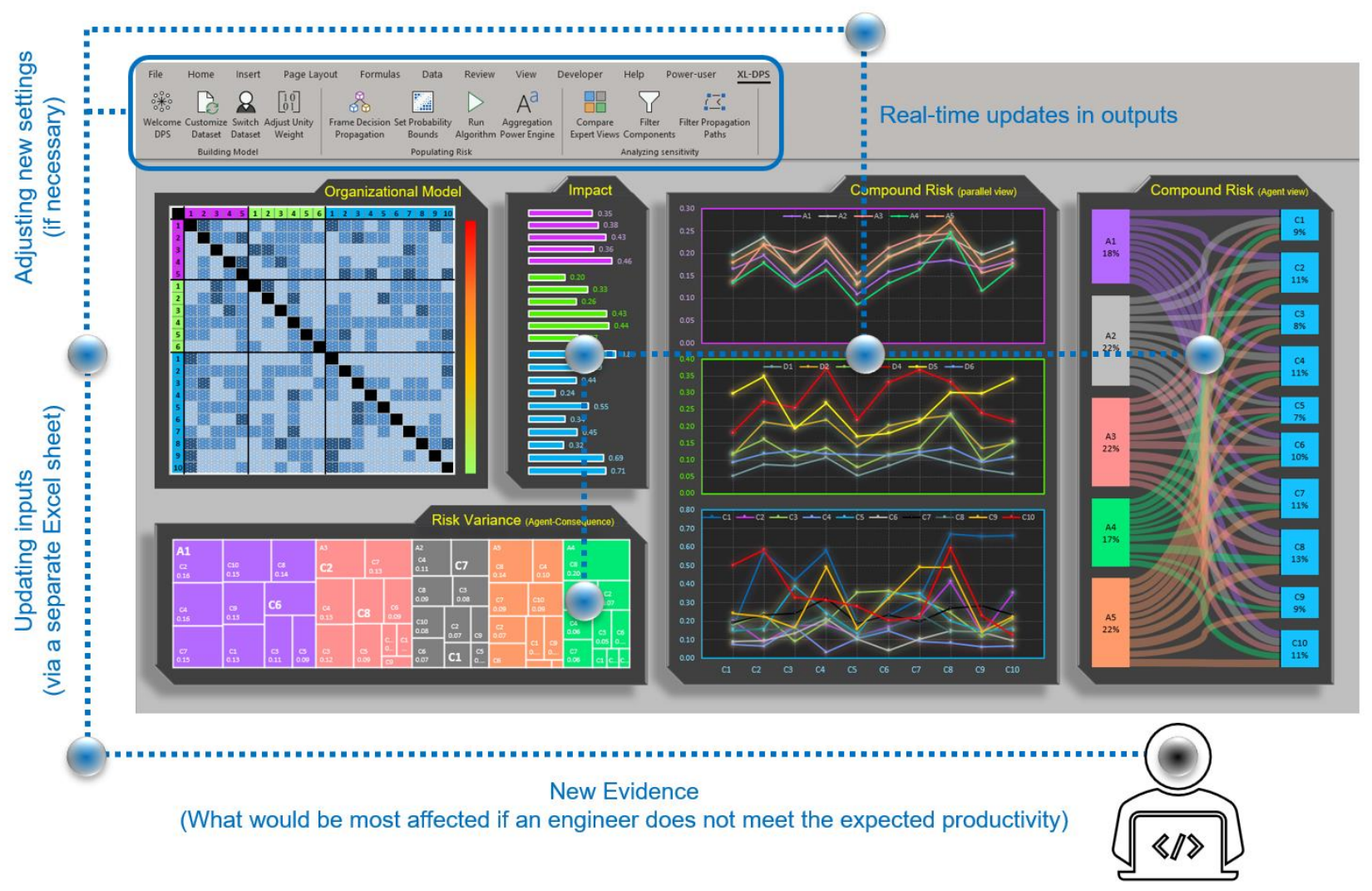

Fig. 9. Screenshot of the DPM prototype interface: interacting with the tool and telling business stories.

\#14 and \#16), the DPM dashboard has been linked to an interactive dataset sheet (to directly store new evidence) and a backup risk computation sheet (with details of computations about underlying change model).

The primary evaluation focused upon a live simulation of the DPM prototype tool and mechanics of the model with reference to the BT's FED case. Reviewing the research questions (Section 2) reflected high face validity of the model [52] owing to the fact that it was built based on an extensive conversation with stakeholders and domain experts on the modeled system, driven by business problem, and applied to data from an industrial setting. Comparing the results with the original study - which was performed using company documents with unity data and used as the baseline for workshops [53] - verified the capability of the model in handling poor-quality and subjective data (Req. \#2).

Furthermore, the primary evaluation shows the effectiveness of the DPM's approach in coping with complexity of a multi-layer dense network and producing risk plots without saturation (Req. \#6), as opposed to the benchmarked algorithms (Fig. 3). The impact of incorporating organizational dynamics into change propagation modelling should not be underestimated, as it is such a big step towards designing a more effective organization through understanding for instance what settings at the decision layer achieve the most efficient cost-benefit trade-off (at the consequence layer), and what are the associated influencers (at the agent layer)? Figure 10 illustrates an example of the compound risk of Engineer role on the Number of SLAs $\left(C R_{A 5 C 8}\right)$, and its underlying computational layers in DPM. From an individual change perspective, the impact bar in Fig. 9 shows Engineer as the most influential role amongst the modelled agents (Req. \#8). By concentrating at the compound likelihood, the network-level aggregation - following (6) - confirms the importance of hidden links between input and output components; in this case, the route involving the mutual influences between agents $\left(A_{5} \rightarrow A \rightarrow D \rightarrow C_{8}\right)$ significantly increases the compound risk (Req. \#12). 


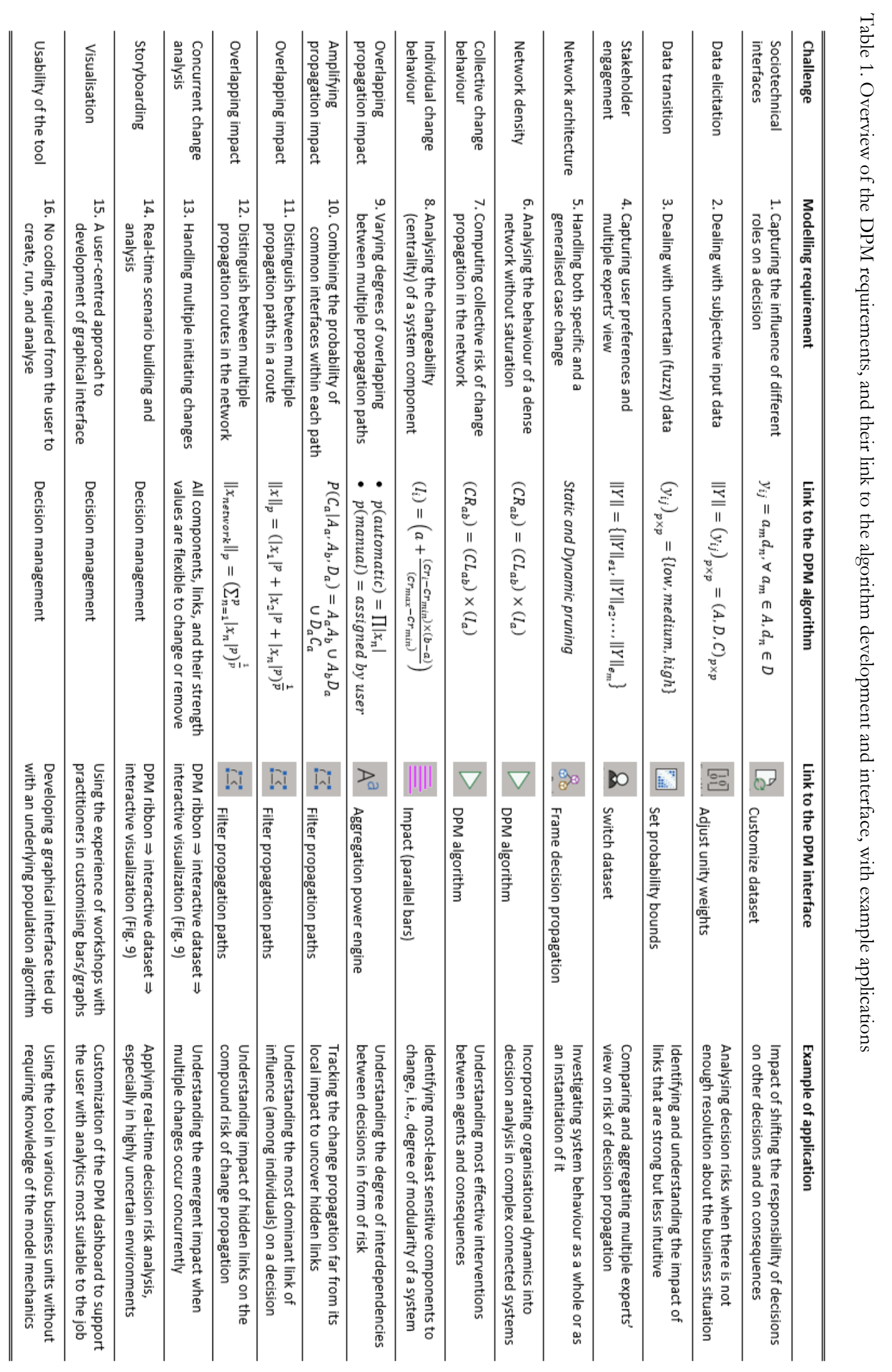


Accordingly, digging into the details of these (dominant) route in Fig. 10(C) can provide further information about the way that multiple paths behave in responding to a change, thus identifying the most and least influential paths (Req. \#11). This collective behavior cannot directly be inferred by looking at the causal pathways in a network. It also verifies the assumptions we previously made on overlapping impact that (1) the impact of multiple propagation paths passing through common individuals - in this example, $A_{4}$ - cannot be considered independently (Sec. 3.1), and (2) the more propagation path in a route, the lower probability of aggregated path and the higher probability that there is a dominant path amongst them (5.4.1(b)); comparing the value of dominant path (0.512) with the dominant route (0.517) proves this assumption.

Similar investigations on other components eventually enabled the workshop participants to trace the propagation analysis back to the links which formed the dominant path $A_{5} \rightarrow A_{4} \rightarrow D_{3} \rightarrow C_{8}$ (Req. \#10), resulting into the identification of key communication and decision links and pathways. It also encouraged them to think about what might possibly go wrong in their daily jobs (Fig. 2) and generate a range of business scenarios to understand what the impact of those changes on other roles and decisions in the organization might be, in an interactive manner.

\subsection{Scenario analysis}

All Upon verifying the simulation model, the focus of the subsequent implementation workshops has largely been on creating and testing a range of what-if business scenarios to explain the model behavior in real settings. Within the overall business problem discussed earlier in Sec. 3 and reflected in Table 1, this section focused upon illustrating how the collective change behavior (Req. \#7) is sensitive to sociotechnical interfaces (Req. \#1) and multiple initiating changes (Req. \#13).

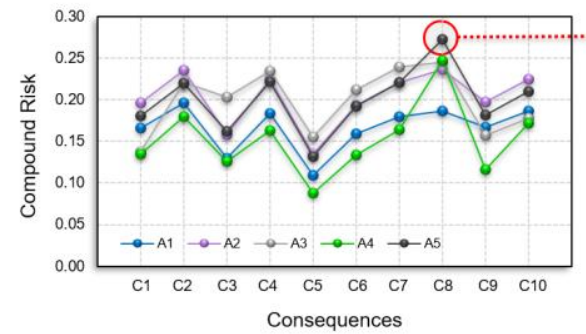

(A) Compound Risk plot (compound view of agent on consequences; screenshot from the DPM dashboard)

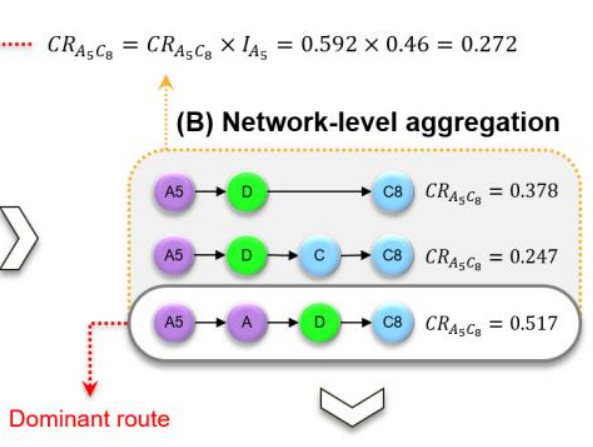

(B) Network-level aggregation

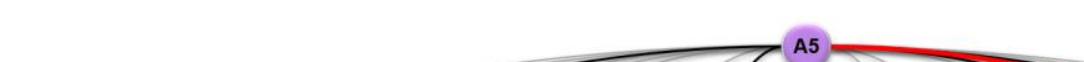

(D) Component-level aggregation

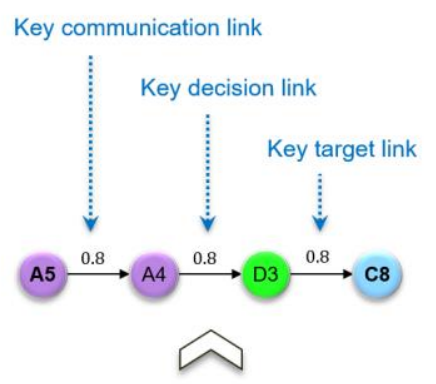

(C) Route-level aggregation

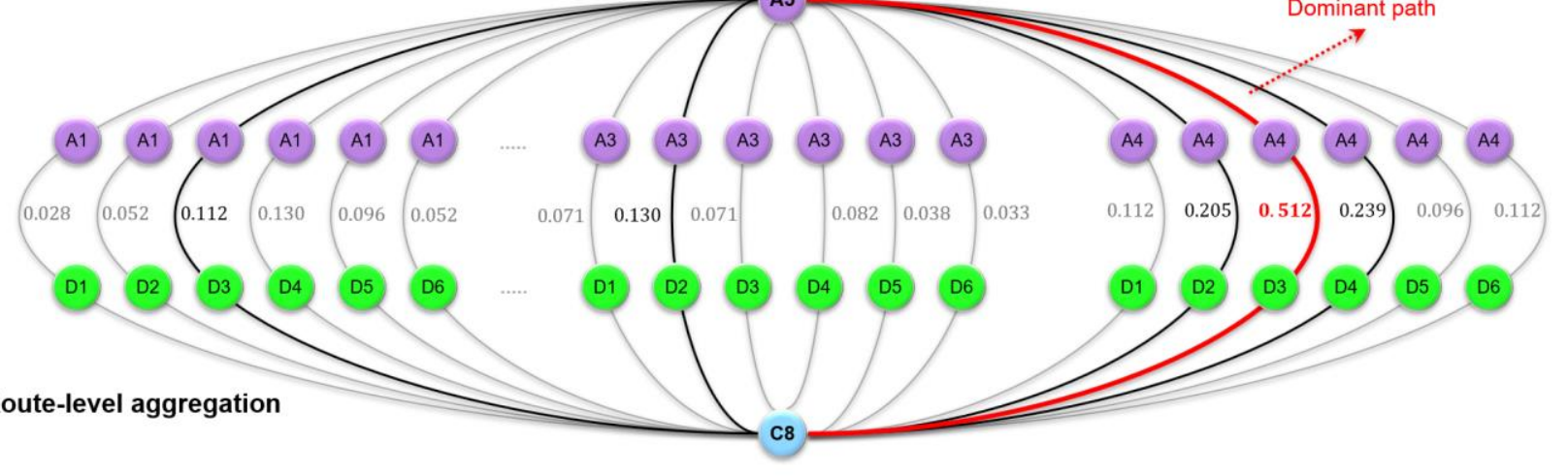

Fig. 10. Compound risk in the DPM algorithm and its underlying computational layers. 
Overall, 14 business narratives generated and examined by the participants (Req. \#14), some of them have been a day-to-day challenge to decision-makers, such as finding the most effective intervention between Deploying Engineers and Productivity Improvement (reprioritizing objectives), or negotiating on how shift in the responsibility of decisions like Deploying Engineers and Performing jobs affect the quantity and quality of these jobs (rethinking responsibilities). These scenarios, illustrated in Fig 11, are discussed in the following to demonstrate usefulness of the DPM as a decision support tool (Req. \#16).

(i) Reprioritizing objectives. An important application of DPM would be helping the Planner (shown as $A_{3}$ in the model) in deploying engineers $\left(D_{2}\right)$ so as to maximize the productivity $\left(C_{4}\right)$ of engineers $\left(A_{2}\right)$. From a change management perspective, this can be examined by improving the link between deploying engineers and productivity $\left(D_{2} C_{4}\right)$ from its current situation low to presumably high. The objective is to understand how this little change can instinctively affect other interlinked decisions and associate agents. From an agent standpoint, the result generally shows a significant increase on the influence of planner (Fig. 11.B1), and also manager and engineer, on employee satisfaction $\left(C_{3}\right)$ and productivity $\left(C_{4}\right)$. From decision view (Fig. 11.B2), this interestingly leads to the increase on the influence of deploying engineers on employee satisfaction $\left(C_{3}\right)$, work-life balance $\left(C_{5}\right)$, and somehow travelling time $\left(C_{6}\right)$; nevertheless, this does not affect key measurable outcomes such as SLAs $\left(C_{8}\right)$ and total cost $\left(C_{9}\right)$. The practitioners found this behavior reasonable, because one way to compensate an under-forecast plan is to ask the available engineers to work overtime, but this contributes to diminish the productivity. An alternative is to improve the productivity of engineers by minimizing the travelling time. If appropriately managed, this can overcome the shortfall while improving the employee satisfaction through minimizing the travelling time - thus avoiding overtime jobs.

(ii) Rethinking responsibilities. Another promising application of DPM would be about understanding the impact of organizational dynamics when making a joint decision. We examined this scenario by applying multiple

A) Original implementation
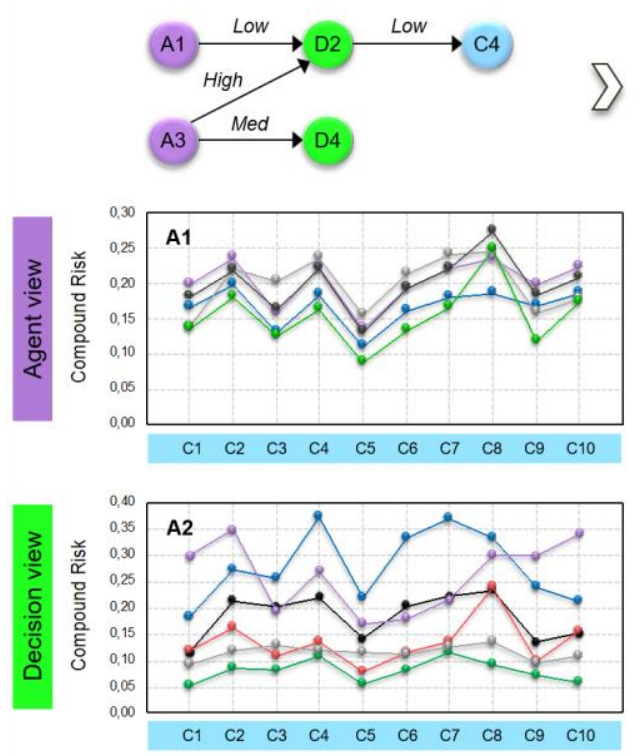

B) Scenario $i$ : Reprioritizing objectives
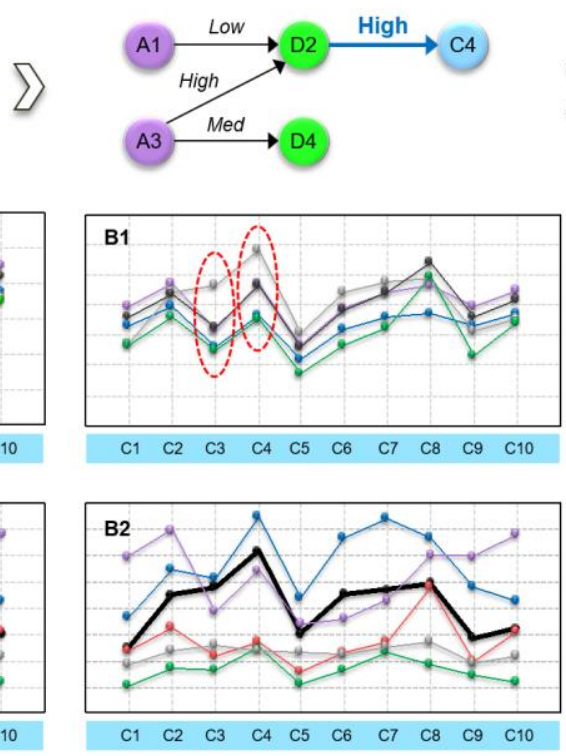

C) Scenario ii: Rethinking responsibilities
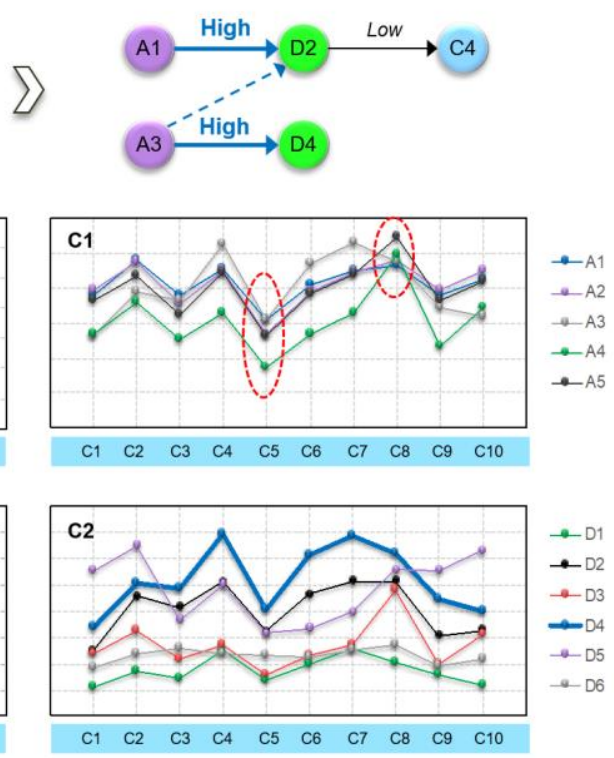

Fig. 11. Representation of Compound Risk on Consequences for different business scenarios. 
changes to decision responsibilities concurrently (Req. \#13): giving the director $\left(A_{1}\right)$ the full responsibility of deploying engineers $\left(A_{1} D_{2}\right.$ to high), while taking out its responsibility from the planner $\left(A_{3} D_{2}\right.$ to zero $)$, and instead giving the planner the full responsibility of performing tasks $\left(A_{3} D_{4}\right.$ to high). The obvious indirect impact of such changes is on the general influence of controller $\left(A_{4}\right)$, in particular over work-life balance $\left(C_{5}\right)$. This is while others' role has been jumped up (Fig. 11.C1). Another key observation is an even increase in the involvement of agents over the number of SLAs. "This looks very interesting; it means every one's influence is important to achieving the desired number of SLAs - said an Operations Field Expert and continues - it is equivalent to less Command and Control which might result into more tension between employees.

From a decision view, the quantity of jobs $\left(D_{4}\right)$ is observed not to be as important as it was previously, whereas the quality of jobs $\left(D_{5}\right)$ has become more important (Fig. 11.C2). This is consistent with the observations on the (increased) influence of engineers and (decreased) role of planner over the SLAs. The quick insight, represented by experts, is that just solely giving the director the opportunity to make this decision $\left(D_{2}\right)$ does not necessarily mean translating it down through the organization unless actually you give those individuals the responsibility to enact.

Similar sort of scenario analysis and follow-up discussion performed in a full-day workshop with domain experts and continued in the last workshop with OpenReach decision-makers to re-evaluate their inputs based on the model behaviour. During these two implementation workshops lasting for about two months, the DPM prototype (along with basic instructions) transferred to the company for building a confidence in using the tool (Req. \#16). Due to the participatory nature of modelling and analysis, it was not found by the authors to be a difficult task to make the practitioners familiar with the core concepts of DPM and discussing the improvement leverages in the last ramp-up workshop.

\section{Discussion and conclusive remarks}

The concept of change propagation has not previously been studied as a purpose for quantifying the desirability of organizational decisions. It is extremely challenging per se, since individuals are reflective and might react (even) to the same situation differently. Understanding of sociotechnical interfaces is additional challenge, where the majority of routine decisions are made through informal communication channels, and knowledge on which would be tacit and highly subjective.

As a response to these challenges in a Telecom company, this research presented the development of an alternative way of capturing, interpreting, and visualizing probabilistic connections in complex organizational systems. The proposed method, referred to as Decision Propagation Method - DPM, is a predictive tool - based on real constraints and data - that is able to proactively support decision-makers on where to make changes in the organization to achieve the desired set of outcomes, e.g., in objectives, priorities, roles? Inspired by requirements in BT, DPM is primarily designed for situations in that there might not be sufficient resolution of the business context such as poor-structured systems and early design phases [54]. It has reflected a proven capability to compute Compound Risk based on both unity and fuzzy input data. The probabilistic organizational model in DPM can be derived from simple causal effect diagrams such as fuzzy cognitive map. This builds a potential capability in DPM - to be considered as future directions - to be mapped from participatory modelling workshops or to be used as a collaborative decision support tool.

In developing the computer support, attention made not only to the quality of analysis, but also efforts put into communication and adoption of the method to ensure its successful implementation in real settings. The implementation of DPM has accommodated the density of connections; and the design of a novel dashboard acted as an interface to the model which enables real-time interaction and decision support. In fulfilling the requirements specified by industry partner (Table 1), DPM presented a measure of risk that combines our understanding of the individuals change behavior (inspired by network science) and collective change behavior (inspired by graph theory). 
Through an extensive case study, it is illustrated that quantifying the collective risk of consequences - when an individual's decision propagates across the system - can support decision-makers to be proactively aware of the consequence of their actions, thus acting as a feedback mechanism to individual roles. In fact, analysis of business scenarios in this paper showed how local change in performance of each role affects the local performance of other roles and dispersion of such propagations influence the entire system.

The evaluation process was not however without obstacles. When some model outputs did not confirm the stakeholders' expectations in scenario building workshop, there was a robust debate about the implications and caution in using such results to inform decision-making. In these situations, it was particularly useful to invite stakeholders to interact with the DPM tool, challenge their assumptions, and test their expectations against model outputs. Eventually, demonstrating to partners that the model assumptions can be far expanded to support numerous real-life business situations confirmed its validity and built confidence for its internal use in the company.

The potential impact of this research to support decision-making is significant - particularly in the light of ever more connected products and manufacturing processes. Not only for engineering and business sectors which often make interconnected decisions, but also for the academic community which evidently have few tools capable of supporting connected decision propagation with overlapping spheres of influence. Such investigation in this paper can open up several possibilities for future research such as (1) investigating the varying degrees of overlapping between propagation paths; (2) taking the directionality (in terms of positivity or negativity) of changes into overlapping computation, i.e., the impact of decision A might be positive on decision B and at the same time, be negative on decision C; (3) studying the relationship between length of propagation and degree of overlapping between propagation paths; and (4) incorporating and aggregating multiple experts' view into decision propagation modelling.

\section{Acknowledgment}

The authors would like to acknowledge the funding received from the British Telecom to undertake this research, and also the extensive assistance of Jonathan Malpass and FED team for their inputs during case study. Some material in this paper was adapted and substantially extended from the earlier works of [53].

\section{References}

[1] M. Hassannezhad and P. Clarkson, "A Normative Approach for Identifying Decision Propagation Paths in Complex Systems," in DESIGN2018, 2018, pp. 1559-1570, doi: 10.21278/idc.2018.0436.

[2] W. Zhang and Q. Ji, “A factorization approach to evaluating simultaneous influence diagrams," IEEE Trans. Syst. Man, Cybern. Part ASystems Humans, vol. 36, no. 4, pp. 746-757, 2006, doi: 10.1109/TSMCA.2005.855753.

[3] C. Eckert, P. Clarkson, and W. Zanker, "Change and customisation in complex engineering domains," Res. Eng. Des., vol. 15, no. 1, pp. 1-21, 2004, doi: 10.1007/s00163-003-0031-7.

[4] E. Koh, N. Caldwell, and P. Clarkson, "A technique to assess the changeability of complex engineering systems," $J$. Eng. Des., vol. 24, no. 7, pp. 477-498, 2013, doi: 10.1080/09544828.2013.769207.

[5] D. Wynn, N. Caldwell, and P. Clarkson, "Predicting Change Propagation in Complex Design Workflows," J. Mech. Des. Trans. ASME, vol. 136, no. 8, p. 081009, 2014, doi: 10.1115/1.4027495.

[6] B. Hamraz, N. Caldwell, and P. Clarkson, "A matrix-calculation-based algorithm for numerical change propagation analysis,” IEEE Trans. Eng. Manag., vol. 60, no. 1, pp. 186-198, 2013, doi: 10.1109/TEM.2012.2203307.

[7] S. Sarica and J. Luo, "An Infinite Regress Model of Design Change Propagation in Complex Systems," IEEE Syst. J., pp. 1-9, 2019, doi: 10.1109/JSYST.2019.2899988.

[8] M. Hassannezhad, S. Cassidy, and P. Clarkson, "Dynamic modelling of relationships in complex service design systems," in ICED'17, 2017, vol. 2, no. DS87-2.

[9] P. Clarkson, C. Simons, and C. Eckert, "Predicting Change Propagation in Complex Design," J. Mech. Des. Trans. ASME, vol. 136, no. August 2014, pp. 1-13, 2004, doi: 10.1115/1.1765117. 
[10] R. Schaffer, “All Management Is Change Management," Harvard Business Review, 2017.

[11] G. Levchuk, Y. Levchuk, J. Luo, K. Pattipati, and D. Kleinman, "Normative design of organizations - Part II: Organizational structure," IEEE Trans. Syst. Man, Cybern. Part ASystems Humans, vol. 32, no. 3, pp. 360-375, 2002, doi: 10.1109/TSMCA.2002.802820.

[12] B. Hamraz, N. Caldwell, and P. Clarkson, "A Holistic Categorization Framework for Literature on Engineering Change Management," Syst. Eng., vol. 16, no. 4, pp. 473-505, 2013, doi: 10.1002/sys.

[13] T. Jarratt, C. Eckert, N. Caldwell, and P. Clarkson, "Engineering change: An overview and perspective on the literature," Res. Eng. Des., vol. 22, pp. 103-124, 2011, doi: 10.1007/s00163-010-0097-y.

[14] A. MacCormack, J. Rusnak, and C. Baldwin, "Exploring the Structure of Complex Software Designs: An Empirical Study of Open Source and Proprietary Code," Manage. Sci., vol. 52, no. 7, pp. 1015-1030, 2006, doi: $10.1287 /$ mnsc. 1060.0552 .

[15] T. Cohen, S. Navathe, and R. Fulton, "C-FAR, change favorable representation," Comput. Des., vol. 32, pp. 321-338, 2000.

[16] D. K. H. Chua and M. a. Hossain, "Predicting Change Propagation and Impact on Design Schedule Due to External Changes," IEEE Trans. Eng. Manag., vol. 59, no. 3, pp. 483-493, Aug. 2012, doi: 10.1109/TEM.2011.2164082.

[17] Y. Li, W. Zhao, and Y. Ma, "A shortest path method for sequential change propagations in complex engineering design processes," Artif. Intell. Eng. Des. Anal. Manuf. AIEDAM, vol. 30, no. 1, pp. 107-121, 2016, doi: 10.1017/S0890060415000311.

[18] I. Ullah, D. Tang, Q. Wang, and L. Yin, "Least Risky Change Propagation Path Analysis in Product Design Process," Syst. Eng., vol. 20, no. 4, pp. 379-391, 2017, doi: 10.1002/sys.21400.

[19] D.-B. Tang, L.-L. Yin, Q. Wang, I. Ullah, H.-H. Zhu, and S. Leng, "Workload-based change propagation analysis in engineering design," Concurr. Eng. Res. Appl., vol. 24, no. 1, pp. 17-34, 2016, doi: 10.1177/1063293X15608510.

[20] M. Sosa, S. Eppinger, and C. Rowles, "A Network Approach to Define Modularity of Components in Complex Products," J. Mech. Des. Trans. ASME, vol. 129, no. 11, p. 1118, 2007, doi: 10.1115/1.2771182.

[21] E. Suh, O. De Weck, and D. Chang, "Flexible product platforms: Framework and case study," Res. Eng. Des., vol. 18, no. 2, pp. 67-89, 2007, doi: 10.1007/s00163-007-0032-z.

[22] M. Giffin, O. De Weck, G. Bounova, R. Keller, C. Eckert, and P. Clarkson, "Change Propagation Analysis in Complex Technical Systems,” J. Mech. Des. Trans. ASME, vol. 131, no. 8, p. 081001, 2009, doi: 10.1115/1.3149847.

[23] S. Collins, J. Bradley, and A. Yassine, "Analyzing Product Development Task Networks to Examine Organizational Change," IEEE Trans. Eng. Manag., vol. 57, no. 3, pp. 513-525, Aug. 2010, doi: 10.1109/TEM.2009.2033047.

[24] P. Hein, B. Morkos, and C. Sen, "Utilizing Node Interference Method and Complex Network Centrality Metrics to Explore Requirement Change Propagation,” in ASME 2017 DETC/CIE, 2017, doi: 10.1115/detc2017-67930.

[25] H. Lee, H. Seol, N. Sung, Y. S. Hong, and Y. Park, "An analytic network process approach to measuring design change impacts in modular products," J. Eng. Des., vol. 21, no. 1, pp. 75-91, 2010, doi: 10.1080/09544820802232517.

[26] S. Li and L. Chen, "Identification of clusters and interfaces for supporting the implementation of change requests," IEEE Trans. Eng. Manag., vol. 61, no. 2, pp. 323-335, 2014, doi: 10.1109/TEM.2013.2292856.

[27] E. Koh, A. Forg, M. Kreimeyer, and M. Lienkamp, "Using engineering change forecast to prioritise component modularisation,” Res. Eng. Des., vol. 26, no. 4, pp. 337-353, 2015, doi: 10.1007/s00163-015-0200-5.

[28] B. Hamraz, N. Caldwell, and P. Clarkson, "A Multidomain Engineering Change Propagation Model to Support Uncertainty Reduction and Risk Management in Design," J. Mech. Des., vol. 134, no. 10, pp. 1-14, 2012, doi: 10.1115/1.4007397.

[29] L. Siddharth and P. Sarkar, "A Multiple-Domain Matrix Support to Capture Rationale for Engineering Design Changes," J. Comput. Inf. Sci. Eng., vol. 18, no. 2, p. 021014, 2018, doi: 10.1115/1.4039850.

[30] M. Ouertani, "Supporting conflict management in collaborative design: An approach to assess engineering change impacts," Comput. Ind., vol. 59, pp. 882-893, Dec. 2008, doi: 10.1016/j.compind.2008.07.010.

[31] N. Ahmad, D. Wynn, and P. Clarkson, "Change impact on a product and its redesign process: A tool for knowledge capture and reuse,” Res. Eng. Des., vol. 24, no. 3, pp. 219-244, 2013, doi: 10.1007/s00163-012-0139-8.

[32] W. Fdhila, C. Indiono, S. Rinderle-Ma, and M. Reichert, "Dealing with change in process choreographies: Design and implementation of propagation algorithms," Inf. Syst., vol. 49, pp. 1-24, 2015, doi: 10.1016/j.is.2014.10.004.

[33] J. Lee and Y. Hong, "Bayesian network approach to change propagation analysis," Res. Eng. Des., vol. 28, no. 4, pp. 437-455, 2017, doi: 10.1007/s00163-017-0252-9.

[34] J. Lee and Y. Hong, "Data-driven prediction of change propagation using Dependency Network," Eng. Appl. Artif. Intell., vol. 70, no. March 2017, pp. 149-158, 2018, doi: 10.1016/j.engappai.2018.02.001. 
[35] H. Cheng and X. Chu, "A network-based assessment approach for change impacts on complex product," J. Intell. Manuf., vol. 23, no. 4, pp. 1419-1431, 2012, doi: 10.1007/s10845-010-0454-8.

[36] M. C. Pasqual and O. L. De Weck, "Multilayer network model for analysis and management of change propagation," Res. Eng. Des., vol. 23, no. 4, pp. 305-328, 2012, doi: 10.1007/s00163-011-0125-6.

[37] S. Jung, O. Asikoglu, and T. W. Simpson, "A method to evaluate direct and indirect design dependencies between components in a product architecture,” Res. Eng. Des., vol. 0, no. 0, p. 0, 2018, doi: 10.1007/s00163-018-0291-x.

[38] C. Rehn et al., "Quantification of changeability level for engineering systems," Syst. Eng., vol. 22, no. 1, pp. 80-94, 2019, doi: 10.1002 /sys.21472.

[39] M. Kivela, A. Arenas, M. Barthelemy, J. Gleeson, Y. Moreno, and M. Porter, "Multilayer networks," J. Complex Networks, vol. 2, no. 3, pp. 203-271, 2014, doi: 10.1093/comnet/cnu016.

[40] A. Ross, D. Rhodes, and D. Hastings, "Defining changeability: Reconciling flexibility, adaptability, scalability, modifiability, and robustness for maintaining system lifecycle value," Syst. Eng., vol. 11, no. 3, pp. 246-262, 2008, doi: 10.1002/sys.20098.

[41] T. Browning, "Design Structure Matrix Extensions and Innovations : A Survey and New Opportunities," IEEE Trans. Eng. Manag., vol. 63, no. 1, pp. 27-52, 2016, doi: 10.1109/TEM.2015.2491283.

[42] G. Q. Huang and K. L. Mak, "Current practices of engineering change management in UK manufacturing industries," Int. J. Oper. Prod. Manag., vol. 19, no. 1, pp. 21-37, Jan. 1999, doi: 10.1108/01443579910244205.

[43] F. Barzegar, M. Hassannezhad, M. Cantamessa, and P. Clarkson, "On the Functionality of Cambridge Advanced Modeller: A Systematic Qualitative-Quantitative Approach,” 2018.

[44] G. A. Ollinger and T. F. Stahovich, "RedesignIT-A Model-Based Tool for Managing Design Changes," J. Mech. Des., vol. 126, no. 2, p. 208, 2004, doi: 10.1115/1.1666888.

[45] V. Kocar and A. Akgunduz, "ADVICE: A virtual environment for Engineering Change Management," Comput. Ind., vol. 61, no. 1, pp. 15-28, 2010, doi: 10.1016/j.compind.2009.05.008.

[46] P. Clarkson and J. Hamilton, “'Signposting', A Parameter-driven Task-based Model of the Design Process,” Res. Eng. Des., vol. 12, pp. 18-38, Jul. 2000, doi: 10.1007/s001630050021.

[47] D. G. Ullman, D. Herling, and B. D'Ambrosio, "What to do next: Using problem status to determine the course of action,” Res. Eng. Des., vol. 9, no. 4, pp. 214-227, 1997, doi: 10.1007/BF01589683.

[48] R. Keller, "Predicting Change Propagation Algorithms Representations Software Tools," 2007.

[49] S.-H. Cha, "Comprehensive survey on distance/similarity measures between probability density functions," City, vol. 1, no. 2, p. 1, 2007.

[50] A. Shirkhorshidi, S. Aghabozorgi, and T. Wah, "A Comparison Study on Similarity and Dissimilarity Measures in Clustering Continuous Data," PLoS One, vol. 10, no. 12, p. e0144059, Dec. 2015, doi: 10.1371/journal.pone.0144059.

[51] M. Kreimeyer, “A Structural Measurement System for Engineering Design Processes," Technical University of Munich, 2009.

[52] R. Smith and J. Morrow, "Product development process modeling," Des. Stud., vol. 20, no. 3, pp. 237-261, May 1999, doi: 10.1016/S0142-694X(98)00018-0.

[53] M. Hassannezhad, S. Cassidy, and P. Clarkson, "Connectivity as the capacity to improve an organization's decisionmaking," in Procedia CIRP, 2019, vol. 84, pp. 231-238, doi: 10.1016/j.procir.2019.04.222.

[54] A. Kusiak and J. Wang, "Dependency Analysis in Constraint Negotiation," IEEE Trans. Syst. Man. Cybern., vol. 25, no. 9, pp. 1301-1313, 1995, doi: 10.1109/21.400508. 